\title{
Status of KM3NeT-ORCA
}

\author{
Thomas Eberl for the KM3NeT Collaboration*广 \\ Friedrich-Alexander University of Erlangen-Nürnberg, \\ Erlangen Centre for Astroparticle Physics \\ E-mail: Thomas.Eberl@physik.uni-erlangen. de
}

\begin{abstract}
The neutrino mass hierarchy can be experimentally determined by a precision measurement of the zenith angle and energy dependent oscillation probabilities of GeV-scale atmospheric electron and muon neutrinos that have traversed the Earth. ORCA (Oscillation Research with Cosmics in the Abyss) is a planned megaton-size water-Cherenkov neutrino detector in the Mediterranean Sea that will employ the deep-sea technology developed for the KM3NeT project to perform this measurement. The key elements of the proposed detector design are outlined and the current status of the ongoing simulation studies is presented. It is shown that the properties of the deep sea water allow for good direction and energy resolutions in both contributing reaction channels, the charged-current interactions of electron and muon neutrinos on the nucleon. The preliminary sensitivity is calculated using detailed detector response functions, but taking into account only a limited number of systematics, and indicates that a median significance of $3 \sigma$ can be reached with about 3 years of data taking.
\end{abstract}

XVI International Workshop on Neutrino Telescopes,

2-6 March 2015

Palazzo Franchetti - Istituto Veneto, Venice, Italy

\footnotetext{
*Speaker.

${ }^{\dagger}$ www.km3net.org
} 


\section{Measuring the neutrino mass hierarchy}

Over the last decades many experiments using solar, atmospheric, reactor and accelerator neutrinos have established beyond doubt that neutrinos undergo flavor oscillations. Most experimental data can be described successfully in a three neutrino type framework in which mass eigenstates with definite and different masses mix into flavor states via three different, relatively large mixing angles and a possible CP-violating phase $\delta$. This is one of the very few observations indicating that the standard model of particle physics is incomplete and thus underlines the importance to understand the questions that remain in neutrino physics. These questions comprise e.g. the absolute mass scale of neutrinos, their Dirac or Majorana nature, the possible violation of CP symmetry in the lepton sector and the ordering of the neutrino mass eigenstates, the so called mass hierarchy, which is central to the investigation and understanding of all the other and has for example far reaching implications for the interpretation of cosmological data [1]. The neutrino mass eigenstates are arranged such that two of the eigenstates have very small mass-squared difference $\Delta m_{i j}^{2}$, whereas the third eigenstate lies at a more distant mass-squared difference. Whether the two closely spaced eigenstates are less (normal hierarchy) or more massive (inverted hierarchy) than the third eigenstate is at present unknown.

The recent precise measurement of a non-zero value for the third mixing angle $\theta_{13}$ [2] has greatly enhanced the interest and activities to determine the neutrino mass hierarchy via neutrino oscillation experiments. Whereas vacuum oscillations are insensitive to the sign of the masssquared difference, neutrino propagation through matter, e.g. through Earth or Sun, modifies the oscillation probabilities such that they become dependent on the sign of $\Delta m_{i j}^{2}$ and are different and opposite for neutrinos and antineutrinos [3, 4]. Accordingly, it has recently been proposed (e.g. in [5]) to employ megaton-size water or ice Cherenkov detectors and to precisely measure the energy and zenith angle dependent oscillation probabilities of atmospheric $v_{e}$ and $v_{\mu}$ after their propagation through the Earth interior. Earth matter-induced effects on the oscillation probabilities are largest for neutrino energies $E_{V} \approx 30 \mathrm{GeV} / \rho\left[\mathrm{g} / \mathrm{cm}^{3}\right]$, where $\rho$ is the matter density, which implies that the relevant neutrino energies range from a few to about $20 \mathrm{GeV}$, depending on the zenith angle of atmospheric neutrinos traversing the Earth with its density profile as detailed in the Preliminary Reference Earth Model [6].

Due to the impossibility to magnetize such large detector volumes in natural media, these detectors cannot determine the charge of the lepton arising in charged-current (CC) neutrino-nucleon interactions and hence cannot measure the oscillation probabilities separately for neutrinos and antineutrinos. A measurable net effect remains, however, as the $v \mathrm{~N}$ and $\bar{v} \mathrm{~N}$ cross sections differ significantly in the relevant energy regime of a few $\mathrm{GeV}, \sigma(v \mathrm{~N}) \approx 2 \sigma(\bar{v} \mathrm{~N})$ [7]. Furthermore, in the interesting energy range above a few $\mathrm{GeV}$ the atmospheric flux of neutrinos is around $10 \%$ larger than that of antineutrinos, see e.g. [8].

The difference in the matter modified oscillation probabilities for normal and inverted hierarchy is more prominent for the muon channel than for the electron channel [5]. However, the discriminating oscillation pattern in the electron channel is much more robust against experimental resolution effects and is therefore expected to dominate the significance obtained from the measurement $[9,10]$. In order to make best use of both channels, the experimental resolutions need to be optimized and muon events have to be distinguished from electron events. It has also been re- 
alized that a measurement of the inelasticity $y=\left(E_{v}-E_{\mu}\right) / E_{v}$ of the neutrino-nucleon interaction can support the distinction between the hierarchies [11].

\section{The ORCA detector and simulation results}

The successful deployment and operation of the ANTARES neutrino telescope [12] has demonstrated the feasibility of performing neutrino studies with large volume detectors in the deep sea. The KM3NeT [13] technology builds upon this experience and improves the cost effectiveness of the design by about a factor four. The 19 downward and 12 upward looking photo-sensors, and the readout electronics are hosted within pressure resistant glass spheres, so called Digital Optical Modules (DOMs, Fig. 1, left, [14]). The DOMs are distributed in three dimensional space along flexible strings, one end of which is fixed to the sea floor and the other end is held close to vertical by a buoy. Fig. 1, right, shows the footprint of the 115 strings on the seafloor. Each string is equipped with 18 DOMs with a distance of $6-12 \mathrm{~m}$. The instrumented volume ranges from about $3.65 \mathrm{Mm}^{3}$ to $7.3 \mathrm{Mm}^{3}$. The inter-DOM distance and the average inter-string distance of about $20 \mathrm{~m}$ are subject to optimization with constraints arising from the deployment. The design provides nanosecond precision on the arrival time of all single photons and the necessary precision on the position (few centimetres) and orientation (few degrees) of the photo-sensors by respective calibration units. The string concept is modular by design thereby facilitating a phased and distributed implementation.
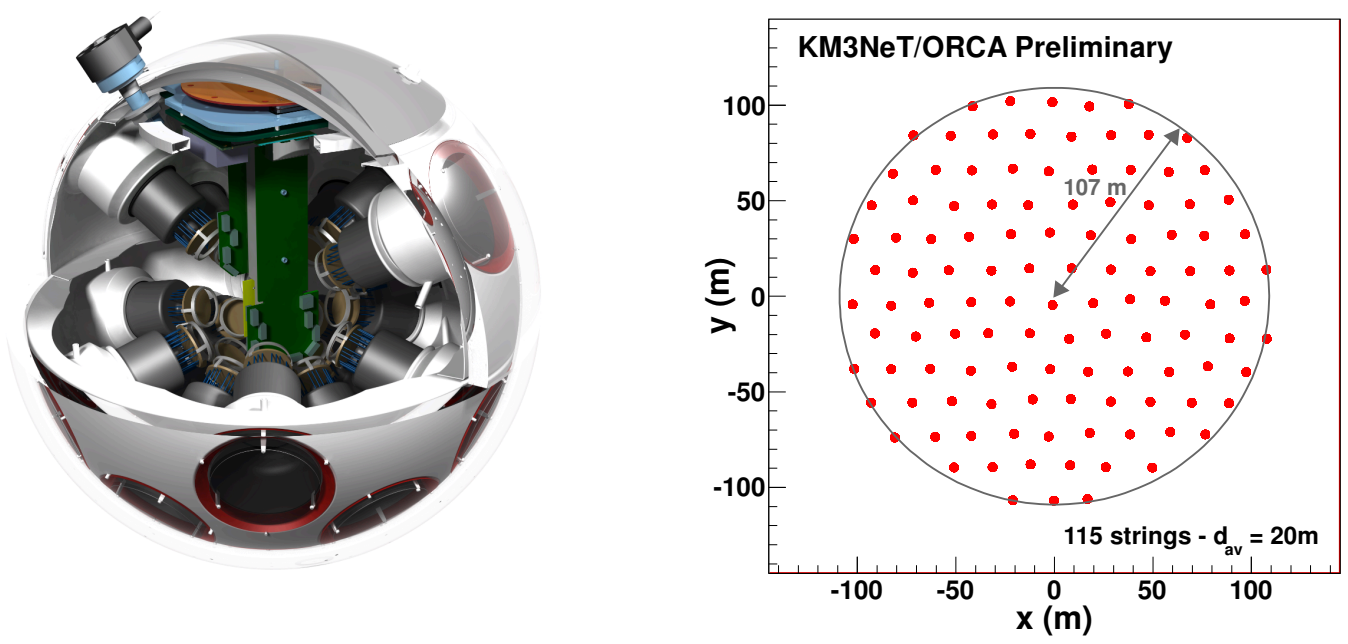

Figure 1: Left: A 3D cutout technical drawing of the DOM. Taken from [14]. Right: Footprint of the ORCA detector (top view), with 115 strings ( $20 \mathrm{~m}$ spacing) with 18 DOMs each ( $6 \mathrm{~m}$ spacing). The instrumented volume is $3.65 \times 10^{6} \mathrm{~m}^{3}$ (cylinder: $R=107 \mathrm{~m}, z=102 \mathrm{~m}$ )

The infrastructure will be located $40 \mathrm{~km}$ offshore from Toulon, France, about $10 \mathrm{~km}$ East of the existing ANTARES telescope, at a depth of $2475 \mathrm{~m}$. The first main electro-optical cable was deployed successfully in December 2014 and the first junction box needed to connect strings has been deployed and connected in spring 2015. Funds have been obtained to build, deploy 
and operate the first 6 strings which is foreseen until the end of 2016. Once more funds become available, the full ORCA array will be constructed and become operational within 3 years.

In order to develop a full event reconstruction chain and determine the detector response functions for the sensitivity calculation, we simulate in detail the particle interactions with the medium surrounding the detector, light generation and propagation as well as its detection by the DOMs. In the simulation chain a volume surrounding the instrumented volume, called "can", is defined. The can volume is a cylinder with height and radius exceeding the instrumented volume by about 3 absorption lengths for the atmospheric muon background simulation and by $40 \mathrm{~m}$ for the neutrino generation. Particles are propagated inside the can and Cherenkov light is generated. Neutrino and antineutrino induced interactions in the energy range from 1 to $100 \mathrm{GeV}$ have been generated with a software package based on the GENIE [15] neutrino event generator. The events are weighted to reproduce the conventional atmospheric neutrino flux following the Bartol atmospheric neutrino flux model [16]. The background due to downgoing atmospheric muons is generated with the MUPAGE [17] program. MUPAGE provides a parameterised description of the underwater flux of atmospheric muons including also multi-muon events. All particles in the can are propagated with a GEANT based software package. It generates Cherenkov light from primary and secondary particles and simulates hits taking into account the light absorption and scattering in water as well as the DOM and PMT characteristics. The optical background arising from the decay of radioactive ${ }^{40} \mathrm{~K}$ in seawater has been taken into account.

Firstly, we describe the results obtained for the reconstruction of events in which an upgoing, i.e. with zenith angle $\theta>90^{\circ}, v_{e}$ reacts with a nucleon via the exchange of a $W$ boson (chargedcurrent reaction). In these events an outgoing electron as well as a hadronic system is created at the vertex. The outgoing electron initiates an electromagnetic shower close to the primary vertex while the hadronic system develops into a hadronic shower with a possibly complex structure of hadronic or electromagnetic subshowers, depending on the decay modes of individual particles in the shower.
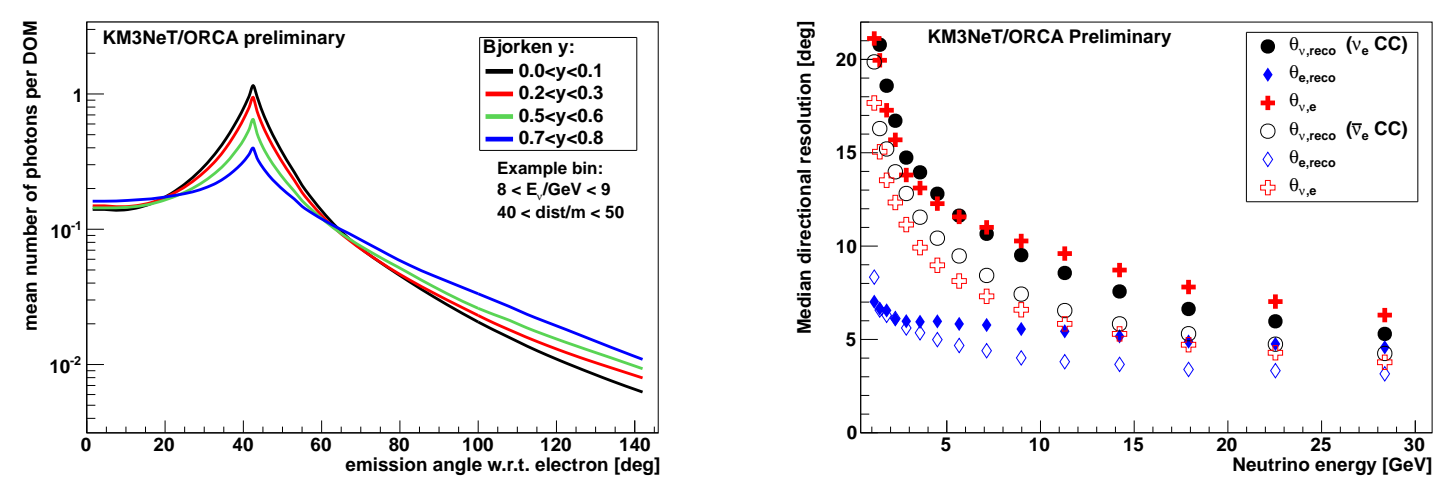

Figure 2: Left: Number of expected photons $\left\langle N_{\gamma}\right\rangle$ as a function of the emission angle $\theta$ between the shower direction and the direction from the vertex to the DOM for different intervals of reaction inelasticity $y$. Right: Median of the kinematic angle between neutrino and electron (red filled crosses), median electron direction resolution (blue filled diamonds) and the median neutrino direction resolution (black filled circles) as a function of neutrino energy for upgoing neutrinos weighted according to the Bartol flux model. Open symbols correspond to the same distributions for $\bar{v}_{e} \mathrm{CC}$ events. 
Fig. 2 (left) shows the distribution of the expected number of photons $\left\langle N_{\gamma}\right\rangle$ on a DOM as a function of the emission angle for different intervals of reaction inelasticity $y$, for shower energies of $8 \mathrm{GeV}<\mathrm{E}<9 \mathrm{GeV}$ and at distances of $40 \mathrm{~m}<\mathrm{d}<50 \mathrm{~m}$.

As the emission angle is defined here with respect to the electron direction, a clear Cherenkov peak of the electron at $42^{\circ}$ is visible. With greater reaction inelasticity and therefore less energetic electron showers this peak is dampened, while the number of expected photons far away from the peak increases due to the brighter hadronic shower. The fact that the angular light profile is preserved, due to the large scattering length of light in water, over distances comparable to the size of the detector and that the mean direction of the hadronic shower at the relevant energies is offset by several degrees, allows in principle to estimate the reaction inelasticity of an event. The resulting discrimination power between neutrino and antineutrino events has not yet been exploited directly in the calculation of ORCA's sensitivity to the mass hierarchy. However, the sensitivity to $y$ is used to improve the energy resolutions as it takes into account the different light yields of electromagnetic and hadronic showers.

The median direction resolutions obtained with the shower reconstruction algorithm are shown in Fig. 2 (right) for the reconstructed electron, the reconstructed electron neutrino and in addition the kinematic angle between the neutrino and the electron. The algorithm clearly reconstructs the electron direction and the resulting uncertainty on the neutrino direction, about 9 degrees at $10 \mathrm{GeV}$ neutrino energy, is dominated by the reaction kinematics of the neutrino interaction. The resolution on the vertex point is on the order of $1 \mathrm{~m}$ or better.

The obtained relative energy resolutions for electron neutrinos and antineutrinos as a function of the neutrino energy are depicted in Fig. 3 (left). The relative energy resolution is defined here as the sigma width of a Gaussian fit over the mean reconstructed energy and we obtain $23 \%$ for $v_{e}$ (red line) with $10 \mathrm{GeV}$ energy. The resolution is better for $\bar{v}_{e} \mathrm{CC}$ events (blue line) than for $v_{e} \mathrm{CC}$ events due to the on average less important light yield from the hadronic shower which also shows large fluctuations.

The effective volume of ORCA to $v_{e} \mathrm{CC}$ events is shown in Fig. 3, right, as a function of neutrino energy and for different zenith angles $\theta$, with $\cos \theta=1$ vertically upgoing neutrinos. The black line shows the effective mass averaged over the zenith angle distribution as given by the Bartol atmospheric neutrino flux model. We obtain an effective mass of about $2.8 \mathrm{Mm}^{3}$ at $10 \mathrm{GeV}$ neutrino energy with differences in zenith angle given by the cylinder geometry and the asymmetric view of the optical modules.

Charged-current reactions of upgoing muon neutrinos with nucleons result in relatively short muon tracks, about $4 \mathrm{~m}$ per GeV of muon energy. The event reconstruction mostly identifies DOMs that have been hit by direct Cherenkov light from the muon and employs a maximum likelihood method to yield the muon track length, the vertex position and a quality parameter. The obtained resolution on the vertex position is better than $4 \mathrm{~m}$ for energies above $5 \mathrm{GeV}$ and the following direction and energy resolutions are reported for events whose vertex point has been reconstructed inside the instrumented volume and whose track fit likelihood fulfills a loose quality criterion. As the algorithm finds the muon with good accuracy and no attempt to account for the hadronic shower is currently made, the angular resolution of the muon neutrino direction reconstruction is completely dominated by the intrinsic contribution, yielding about 7 degrees at $10 \mathrm{GeV}$ neutrino energy as resolution on the neutrino zenith angle, cf. Fig. 4, left. 

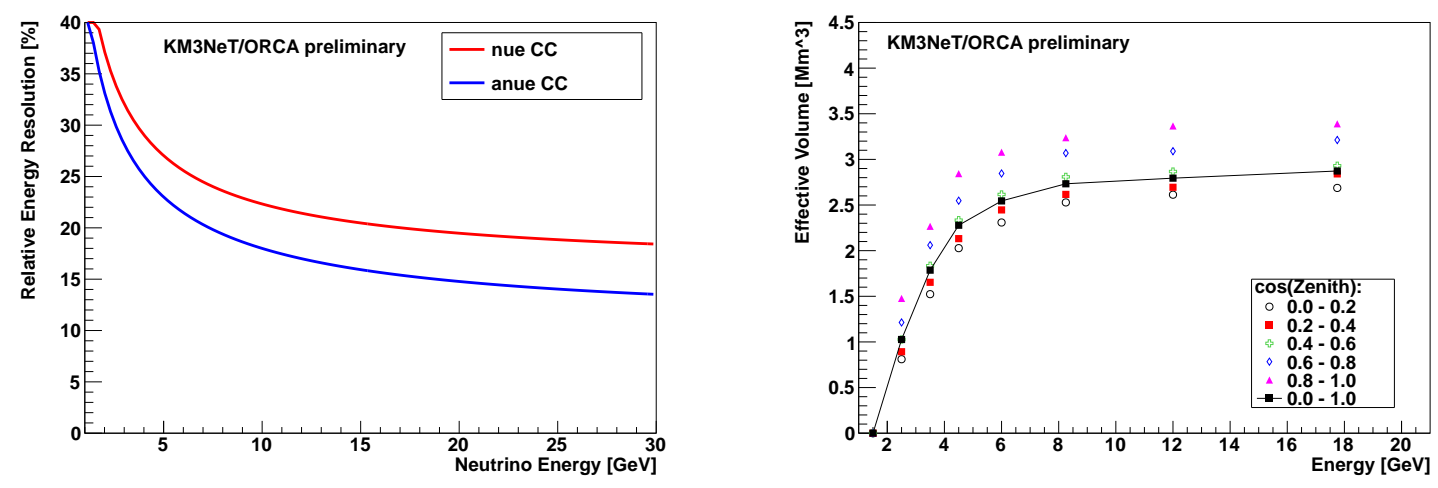

Figure 3: Left: Energy resolution for electron neutrinos (red line) and electron antineutrinos (blue line) as a function of energy. Right: Effective volume for electron neutrinos as a function of neutrino energy for different zenith angles.
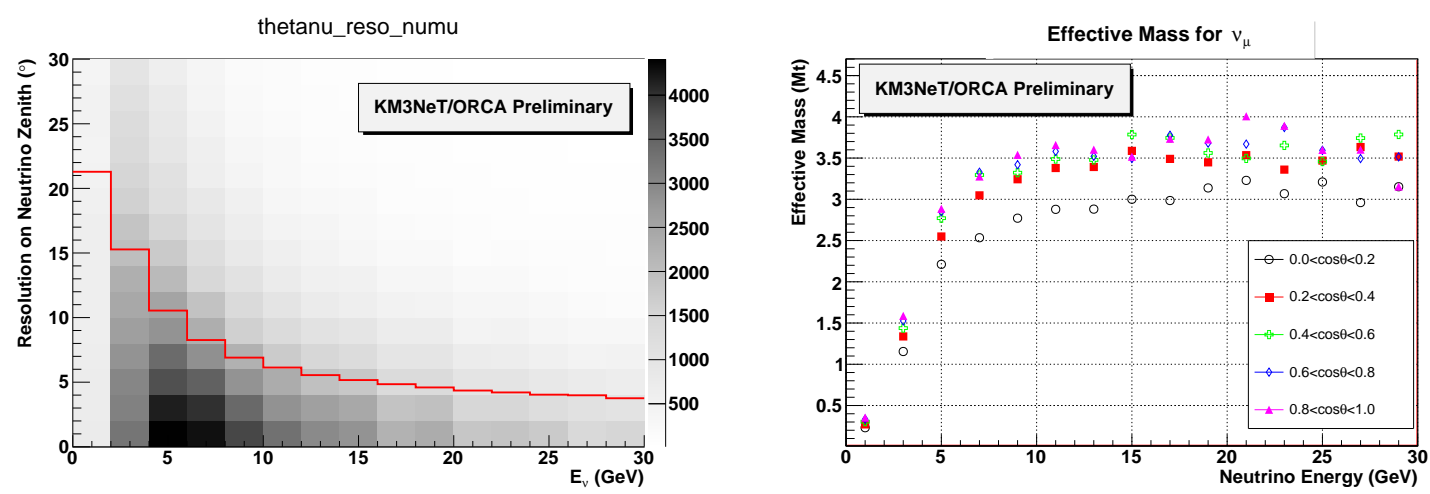

Figure 4: Left: Resolution on the muon neutrino zenith angle as a function of energy. The red line indicates the median of the distribution. Right: Effective mass for $v_{\mu} \mathrm{CC}$ events as a function of energy and for different zenith angle bins. The density of seawater is assumed to be $1.025 \mathrm{~g} / \mathrm{cm}^{3}$.

The energy estimate is mainly based on the reconstructed muon track length, the number of photon hits on DOMs selected by the track recosntruction and their time residuals, i.e. the difference between their expected and measured arrival time. We obtain a fractional energy resolution, defined as $\left|E_{v}-E_{\text {rec }}\right| / E_{v}$, of about $28 \%$ at $10 \mathrm{GeV}$ neutrino energy which improves by about $10 \%$ in the energy range from $5 \mathrm{GeV}$ to $25 \mathrm{GeV}$.

The resulting effective mass for muon neutrinos is depicted in Fig. 4, right, as a function of energy and for different zenith angles. The plateau of the distributon is reached at $10 \mathrm{GeV}$ neutrino energy and on average almost reaches up to the instrumented water mass.

In the Earth traversing atmospheric neutrino flux both muon and electron neutrinos are present and both carry an imprint of the matter-induced oscillation effects that give a handle to determine the mass hierarchy. Therefore it is important to distinguish the different flavors interacting in the detector and to reconstruct and analyse the two channels separately. To this end we have chosen to employ a machine learning algorithm, a random decision forest (RDF, [18]), that is trained on Monte Carlo event data to distinguish between two classes, track-like and shower-like events. A RDF consists of many decision trees that individually categorize an event into different classes and 
whose decisions are combined into a majority vote with a configurable threshold. The algorithm relies on several discriminating observables that combine information extracted from the event. The results of the above described reconstruction algorithms, in particular the fit qualities, are key to the correct classification of a shower-like $v_{e}$ or track-like $v_{\mu}$ event. The fraction of correctly classified events of a certain class is defined as the ratio between number of correctly classified events of this class and the number of all such events in the classified sample. This quantity is shown in Fig. 5, left, as a function of neutrino energy for different neutrino flavors and reaction channels. Muon neutrino charged-current reactions are most often classified correctly as track-like, as the resulting muon will have the highest energy and therefore longest track at a given neutrino energy. Neutralcurrent (NC) events are easier to recognize as shower-like than $v_{e} \mathrm{CC}$ events, as the bright and collimated electromagnetic cascade induced by the electron can be confused with a short muon track. For neutrino energies above $10 \mathrm{GeV}$, the classification is succesful in at least $80 \%$ of the events.
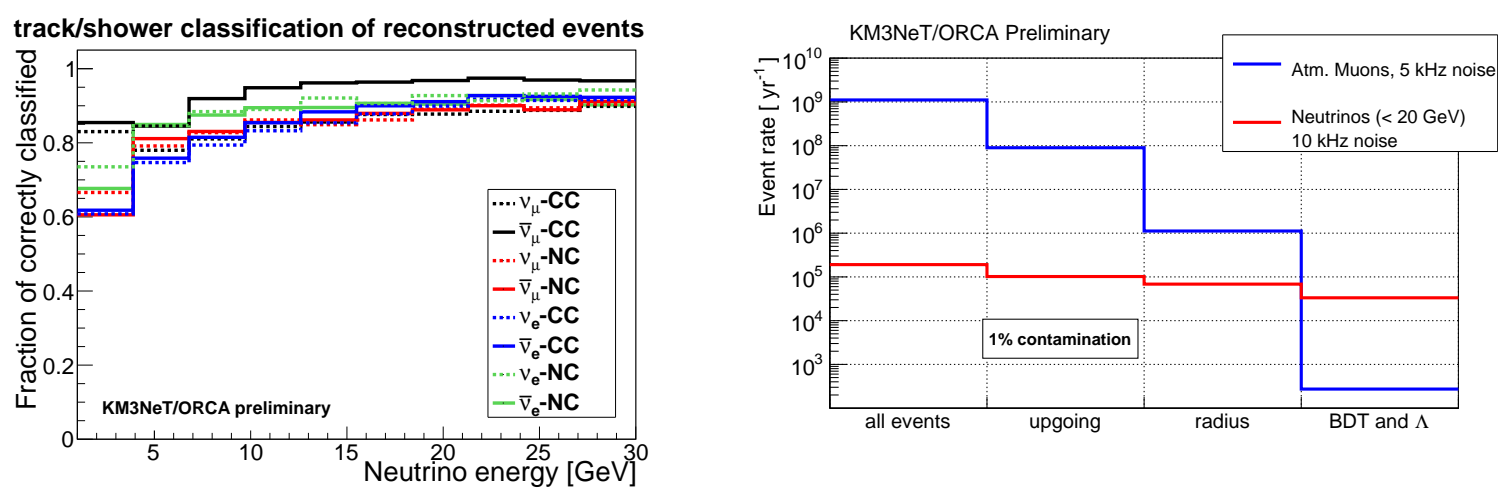

Figure 5: Left: Fraction of correctly classified events as function of the neutrino energy. The classification is trained to distinguish between two classes, shower-like and track-like events. Right: Summary of the cumulative atmospheric muon rejection cuts and their efficiency.

Muon bundles that are created in the atmosphere and enter the detector volume from above constitute a particle background which is $\mathrm{O}\left(10^{6}\right)$ times more abundant than the upgoing atmospheric neutrino flux that is targeted by the measurement. As we derive from our simulation study, the resolution of the muon zenith angle reconstruction is on the order of a few degrees. However, selecting muon tracks according to their reconstructed zenith angle as upgoing, still yields a contribution of misreconstructed seemingly upgoing atmospheric muons that present a too high background for the measurement. Consequently, a multi-step algorithm has been developed to recognize and reject these misreconstructed events. After a loose cut on the quality of the track fit and the estimated reconstruction error, a track starting point is calculated for the event. It is observed that this vertex point is found inside the detector volume or very close to the outermost strings for neutrinos while the fit yields a pseudo-vertex which is far outside the instrumented volume for atmospheric throughgoing muon tracks, which suggests a cut on the radial distance of the pseudovertex from the detector centre. Fig. 5, right, summarizes the individual effect of the different steps of the muon rejection algorithm for atmospheric muons (blue line) and neutrinos (red line). The last cut, labelled BDT, employs a machine learning algorithm, a boosted decision tree, to combine information on the reconstructed direction, the track quality and the pseudo-vertex and results in 
the best muon suppression. In Fig. 5, the cut on the machine learning algorithm output was chosen such that a muon contamination of $1 \%$ remains in the reconstructed neutrino sample while $>60 \%$ of the neutrinos can be retained.

\section{Sensitivity calculation}

Building on the obtained simulation results presented above the sensitivity of ORCA to the mass hierachy is estimated. The employed procedure to calculate the median sensitivity as a function of time, taking into account all detector dependent and independent inputs is explained in the following.

ORCA will determine electron and muon neutrino event rates as a function of the reconstructed energy and zenith angle. By comparing these to the expected distributions a distinction between the two mass hierarchy cases is achieved. The computation of the rate expectations is separated into two parts. Firstly, the expected neutrino interaction rate at the detector site is calculated as a function of the true neutrino energy and zenith angle. Secondly, the response of the detector is modelled resulting in event rates as a function of the reconstructed energy and zenith angle. For the first step, the atmospheric neutrino fluxes are taken from [16] using the tabulated values for the Frejus site, which are similar to the ORCA detector site. The oscillation probabilities depend on the mixing parameters (including the hierarchy) and the density profile of the Earth. They are calculated by evaluating the time evolution operator for neutrino propagation in a constant density medium [20] at small steps along the trajectory. The employed density profile of the Earth corresponds to the Preliminary Reference Earth Model. The CC and NC neutrino-nucleon crosssections from the GENIE Monte Carlo generator for reactions on protons and oxygen nuclei are used. The sensitivity to the mass hierarchy is estimated using likelihood ratios based on pseudoexperiments. For these the energy- and zenith angle-dependent effective volume for muon and electron neutrinos is used to calculate the reconstruction rate in the detector and here background contributions due to remaining misreconstructed atmospheric muons are taken into account. In a further step the particle identification algorithm described above is applied and all events are classified as either track-like or cascade-like.

Finally, angular and energy resolutions as derived from the track and cascade reconstruction algorithms are applied as Gaussian uncertainties. First, the zenith angle is smeared using a threedimensional response matrix that provides binned distributions of $\cos \theta_{\text {rec }}$ as a function of $\cos \theta_{\text {true }}$ and $E_{\text {true }}$. In a second step a two-dimensional response matrix relating $E_{\text {rec }}$ to $E_{\text {true }}$ is used to smear the energy. The resolutions are evaluated separately for each of the event classes.

For each pseudo-experiment a set of uncorrelated, Gaussian-distributed oscillation parameters ( $\Delta M^{2}$ and $\theta_{23}$ ) and a set of systematics, in the following the overall neutrino flux normalization, are chosen and the likelihood is maximised once assuming normal, and once assuming inverted hierarchy to be the true one. The separation between the distributions of the likelihood ratio assuming normal and inverted hierarchy serves as a measure to calculate the significance of the distinction. As a measure of sensitivity, in turn, we use the median significance, i.e. the significance with which the wrong hierarchy can be excluded when the measured likelihood ratio is at the median of the true hierarchy distribution. Fixing the CP-phase to zero, Fig.6 shows the median significance as a function of time in years of operation of the ORCA detector. The red and blue lines distinguish 


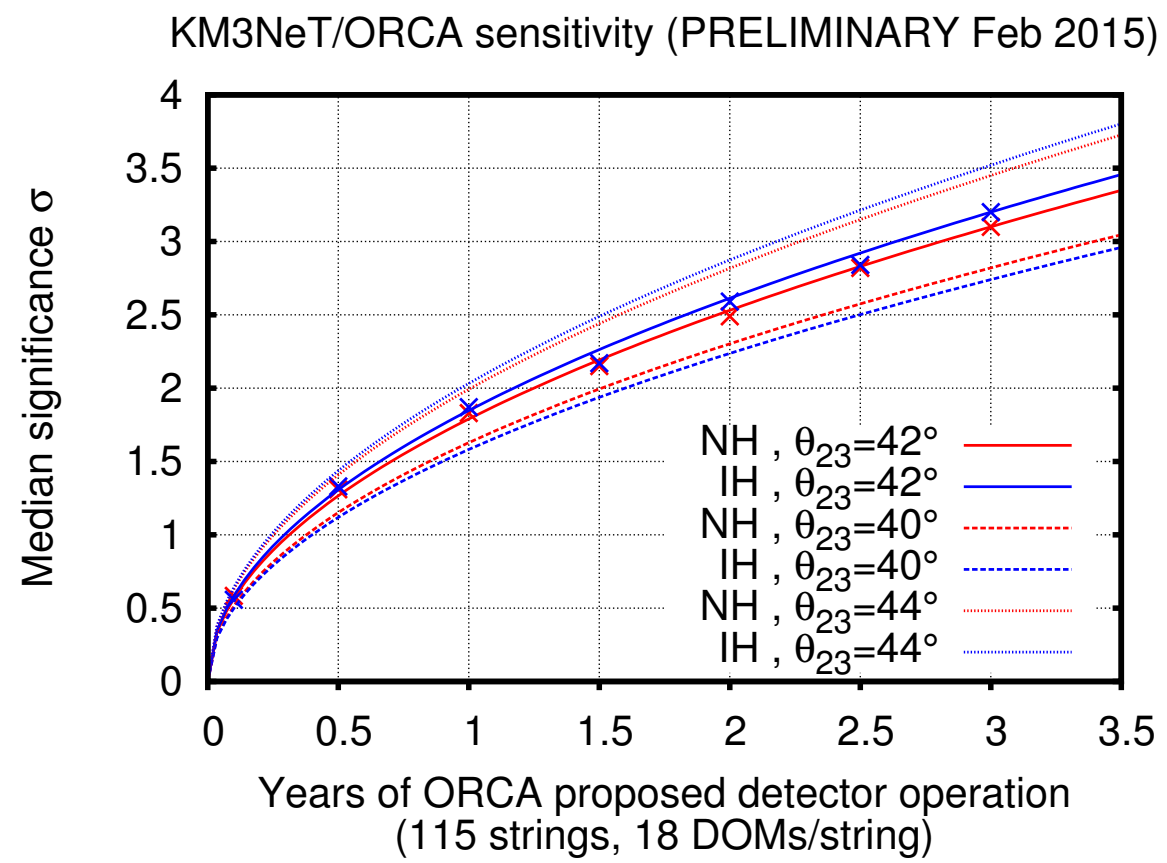

Figure 6: Median sensitivity of ORCA for true normal (red line) or true inverted (blue line) hierarchy and for different true values of the atmospheric mixing angle $\theta_{23}$ as a function of data taking time in years.

between the normal and inverted hierarchy to be the true one for different values (constrained with a Gaussian prior of 2 degrees) of the atmospheric mixing angle $\theta_{23}$. The central two lines correspond to the current global best fit value of $\theta_{23}$ in the first octant, the uncertainty on the sensitivity induced due to the mixing angle $\theta_{23}$ is illustrated by the lines above and below which have been obtained for the current uncertainty on this parameter. A median significance of 3 sigma in 3 years seems attainable with ORCA. Due to the high sensitivity of the experiment to the atmospheric mixing parameters, the mixing angle $\theta_{23}$ and the large mass-squared difference $\Delta M^{2}$, these can be also constrained by ORCA. When these are evaluated as unconstrained free parameters in the likelihood maximisation, we find that after three years of data taking the obtained precision for $\theta_{23}$ is on the order of $0.5^{\circ}$ and $6 \cdot 10^{-5} \mathrm{eV}^{2}$ for $\Delta M^{2}$, which would improve the present uncertainties by roughly a factor 2 .

\section{Conclusions and Outlook}

The ORCA experiment aims at measuring matter-induced modifications of neutrino flavor oscillation probabilities by the detection of atmospheric muon and electron neutrinos that have traversed the Earth interior. Small differences in the zenith angle and energy dependent count rates open the possibility to determine the neutrino mass hierarchy. Detailed detector simulations show that the long scattering length of light in water allows to attain promising direction and energy resolutions for both channels and indicate sensitivity to the reaction inelasticity which will be exploited in further studies. Employing detailed detector response functions for both signal and background, the median sensitivity is estimated to about $3 \sigma$ in 3 years assuming the current best fit value for 
the mixing angle $\theta_{23}$ in the first octant. The inclusion of further systematic uncertainties is under study. In a first construction phase, the deep-sea neutrino telescope technology developed in the KM3NeT project will be employed to construct and operate a densely instrumented 6 string detector off the coast of Toulon, France. It will serve to demonstrate the feasibility of the measurement and to validate and optimize the detector design.

\section{References}

[1] X. Qian and P. Vogel, Neutrino Mass Hierarchy. Preprint arXiv:1505.01891v1 [hep-ex], 2015

[2] F. P. An et al., Daya Bay Collaboration, Chin. Phys. C37, 011001, 2013; J. K. Ahn et al., RENO Collaboration, Phys. Rev. Lett. 108, 191802, 2012

[3] L. Wolfenstein, Neutrino oscillations in matter. Phys. Rev. D17:2369, 1978

[4] S. P. Mikheev and A. Y. Smirnov, Resonant amplification of neutrino oscillations in matter and solar neutrino spectroscopy. Nuovo Cim. C9:17-26, 1986

[5] E. Kh. Akhmedov, S. Razzaque and A. Yu. Smirnov, Mass hierarchy, 2-3 mixing and CP-phase with huge atmospheric neutrino detectors JHEP 1302, 082, 2013

[6] A. M. Dziewonski and D. L. Anderson, Preliminary reference Earth model. Physics of Earth and Planet Interiors 25, 297, 1981

[7] J. Formaggio and G. Zeller, From eV to Eev: Neutrino cross sections across energy scales. Rev. Mod. Phys. 84 (3), 2012

[8] T. K. Gaisser and M. Honda, Flux of atmospheric neutrinos. Ann. Rev. Nucl. Part. Sci. 52, 153, 2002

[9] S. Ge and K. Hagiwara, Physics reach of atmospheric neutrino measurements at PINGU. JHEP 1409, 024,2014

[10] M. G. Aartsen, IceCube-PINGU Collaboration, Letter of Intent: The Precision IceCube Next Generation Upgrade (PINGU). Preprint arXiv:1401:2046 [physics.ins-det], 2014

[11] M. Ribordy and A. Yu. Smirnov, Improving he neutrino mass hierarchy identification with inelasticity measurement in PINGU and ORCA. Phys. Rev. D87, 113007, 2013

[12] M. Ageron et al., ANTARES: the first undersea neutrino telescope. NIM A 656, 2011

[13] M. de Jong, for the KM3NeT Collaboration, KM3NeT perspectives. These proceedings, 2015

[14] S. Adrian-Martinez et al., KM3NeT Collaboration, Deep sea tests of a prototype of the KM3NeT digital optical module. EPJ C74:3056, 2014

[15] C. Andreopoulos et al., The GENIE Neutrino Monte Carlo Generator. NIM A614, 2010

[16] G. D. Barr et al., Phys. Rev. D70, 023006, 2004

[17] G. Carminati et al., An update of the generator of atmospheric muons from parametric formulas (MUPAGE). Comp. Phys. Comm. 181, 2010

[18] L. Breiman, Machine Learning 45, 5-32, 2001

[19] M. Honda et al., Phys. Rev. D83, 123001, 2011, tables available at: http://www.icrr.u-tokyo.ac.jp/ mhonda/

[20] T. Ohlsson and H. Snellman, J. Math. Phys. 41, 2768, 2000, [Erratum-ibid. 42 (2001) 2345] 